\title{
Reconstruction of UWB Impulse Train by Parallel Sampling of Cascaded Identical RC Filters
}

\author{
Hannu Olkkonen ${ }^{1}$, Simo Ahtiainen ${ }^{1}$, Kari Jarvinen ${ }^{1}$, Juuso T. Olkkonen ${ }^{2}$ \\ ${ }^{1}$ Department of Applied Physics, University of Eastern Finland, Kuopio, Finland; ${ }^{2}$ VTT Technical Research Centre of Finland, Espoo, \\ Finland. \\ Email: hannu.olkkonen@uef.fi
}

Received January $19^{\text {th }}, 2013$; revised March $4^{\text {th }}, 2013$; accepted March $18^{\text {th }}, 2013$

Copyright (C) 2013 Hannu Olkkonen et al. This is an open access article distributed under the Creative Commons Attribution License, which permits unrestricted use, distribution, and reproduction in any medium, provided the original work is properly cited.

\begin{abstract}
In this framework we present a new method for measurement of the UWB impulse train based on the parallel sampling of the cascaded identical RC filters. We show that the amplitudes and time locations of $p$ sequential impulses can be reconstructed from simultaneous measurement of the outputs from $2 p$ cascaded identical RC filters. The parallel sampling scheme has a wide range of applications including the detection of the ultra wideband (UWB) impulses. Due to identical analog RC filters and buffer amplifiers, the parallel sampling scheme is flexible to implement in VLSI applications.
\end{abstract}

Keywords: Impulse Train; Pulse Sequence; UWB; SVD

\section{Introduction}

The conventional sampling methods are based on the sequential measurement of signals at equidistant intervals. If the signal contains abrupt changes, such as edges, impulses and other discontinuities, the signal bandwidth is not limited below the Nyquist frequency and the Shannon's sampling theorem is not warranted. Recently the problem to measure and reproduce non-band limited signals has been widely studied in signal processing society. Excellent articles [1-8] concern on the prefiltering and reconstruction of signals under nonideal sampling constraints. The sampling scheme based on the finite rate of innovation (FRI) recovers a variety of transient signal families, which are not band limited, such as impulses, piecewise linear and amplitude modulated edges.

The measurement and recovery of short term transient signals (Diracs) plays a main role in wireless ultra wideband (UWB) technology, where the information is coded to impulse sequences. The Diracs are fed to an analog circuit, which has a specific impulse response. The purpose of the sampling filter is to lengthen the UWB impulses for equidistant sampling by an analog-to-digital converter. Various methods have been developed for reconstruction of the original signal from discrete samples.

Our research group has introduced a sampling scheme, where the signal is fed to the parallel RC filters, whose outputs are sampled simultaneously $[9,10]$. A variant of the parallel sampling scheme is tailored for detection of edges $[11,12]$. The parallel sampling scheme can be applied to reproduce transient signals without ad hoc knowledge of the signal waveform [13]. Recently a multichannel sampling (MCS) arrangement was introduced, where the input signal is modulated by a set of sinusoidal waveforms, followed by a bank of integrators [14]. The MCS yields Fourier series coefficients, which enable the reconstruction of the input signal.

In this framework we introduce a new method for measurement and reconstruction of the impulse trains based on the parallel sampling of the identical cascaded RC filters. We show that the amplitudes and locations of $p$ Diracs can be reproduced from simultaneous measurements of $2 p$ outputs of cascaded identical RC filters. We compare the reconstruction performance of the present method with the MCS arrangement using an analog electronic circuit simulator.

\section{Theoretical Considerations}

\subsection{Sampling of the Impulse Train}

We consider the impulse train consisting of $p$ sequential Dirac distributions

$$
I(t)=\sum_{i=1}^{p} A_{i} \delta\left(t-t_{i}\right)
$$


where $A_{i}$ are amplitudes and $t_{i}$ the time locations. The impulse train is fed to the $N$ identical cascaded $R C$ filters (Figure 1), each having the impulse response

$$
h(t)=\alpha \mathrm{e}^{-\alpha t} u(t)
$$

$y_{k}(t)(k=0,1, \cdots, N-1)$ of the cascaded RC filter network are obtained as where the unit step function $u(t)$ $=1$ for $t \geq 0$ and $u(t)=0$ for $t<0$ and $\alpha=1 / R C$. The output signals

$$
y_{k}(t)=I(t) * \underbrace{h(t) * \cdots * h(t)}_{(k+1) \text { times }}
$$

In Laplace transform domain we have

$$
Y_{k}(s)=I(s) \underbrace{H(s) \cdots H(s)}_{(k+1) \text { times }}=I(s)\left(\frac{\alpha}{s+\alpha}\right)^{k+1}
$$

The inverse Laplace transform then gives

$$
\begin{aligned}
y_{k}(t) & =I(t) * \frac{\alpha^{k+1}}{k !} t^{k} \mathrm{e}^{-\alpha t} u(t) \\
& =I(t) * C_{k} t^{k} \mathrm{e}^{-\alpha t} u(t)
\end{aligned}
$$

where $C_{k}=\alpha^{k+1} / k$ !. Now we can write the convolution integral

$$
\begin{aligned}
y_{k}(t) & =\int_{0}^{t} \sum_{i=1}^{p} A_{i} \delta\left(\tau-t_{i}\right) C_{k}(t-\tau)^{k} \mathrm{e}^{-\alpha(t-\tau)} u(t-\tau) \mathrm{d} \tau \\
& =C_{k} \sum_{i=1}^{p} A_{i}\left(t-t_{i}\right)^{k} \mathrm{e}^{-\alpha\left(t-t_{i}\right)} u\left(t-t_{i}\right)
\end{aligned}
$$

The prominent idea in this work is to measure the RC filter outputs $y_{k}(t)$ simultaneously at a time

$t_{0}>t_{i}(i=1,2, \cdots, p)$. By denoting the time difference $\Delta_{i}=t_{0}-t_{i}$ and rearranging we obtain

$$
u_{k}=\sum_{i=1}^{p} a_{i} \Delta_{i}^{k}
$$

where the notations $u_{k}=y_{k}\left(t_{0}\right) / C_{k}$ and $a_{i}=A_{i} \mathrm{e}^{-\alpha \Delta_{i}}$ are used.

\subsection{Reconstruction Algorithm}

In the following we develop a reconstruction algorithm for the amplitudes and time locations of the Diracs based

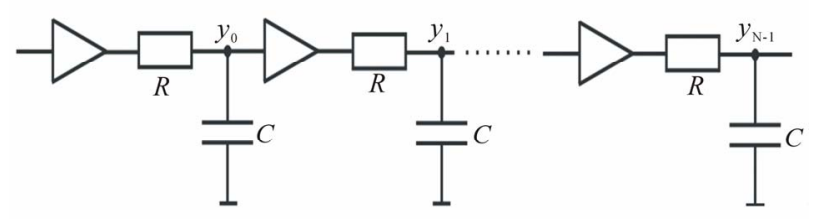

Figure 1. The arrangement for measurement of the impulse train based on the parallel sampling of $N$ cascaded identical RC filters, which are separated by the unity gain buffer amplifiers. on formulation (7). The $z$ transform of the $u_{k}$ sequence with respect to $k$ yields

$$
\begin{aligned}
Z\left\{u_{k}\right\} & =\sum_{k=0}^{\infty} \sum_{i=1}^{p} a_{i} \Delta_{i}^{k} z^{-k}=\sum_{i=1}^{p} a_{i} \sum_{k=0}^{\infty}\left(\Delta_{i} z^{-1}\right)^{k} \\
& =\sum_{i=1}^{p} \frac{a_{i}}{1-\Delta_{i} z^{-1}}
\end{aligned}
$$

Let us define the pole cancellation filter as

$$
H_{p c}(z)=1+\sum_{n=1}^{p} h_{n} z^{-n}=\prod_{j=1}^{p}\left(1-\Delta_{j} z^{-1}\right)
$$

and the $P(z)$ polynomial as

$$
\begin{aligned}
P(z) & =Z\left\{u_{k}\right\} H_{p c}(z) \\
& =\sum_{i=1}^{p} \frac{a_{i}}{1-\Delta_{i} z^{-1}} \prod_{j=1}^{p}\left(1-\Delta_{j} z^{-1}\right) \\
& =\sum_{i=1}^{p} a_{i} \prod_{\substack{j=1 \\
j \neq ?}}^{p}\left(1-\Delta_{j} z^{-1}\right)
\end{aligned}
$$

Clearly the roots of the pole cancellation filter correspond to the roots of the $P(z)$ polynomial. The impulse response $p_{n}(n=0,1, \cdots)$ of the $P(z)$ can be computed from convolution

$$
p_{n}=\sum_{k=0}^{p} u_{n-k} h_{k}
$$

The roots of the $P(z)$ are obtained by setting $p_{n}=0$ for $n \geq 0$. This yields the matrix/vector equation

$$
\begin{aligned}
& {\left[\begin{array}{c}
u_{2 p-1} \\
u_{2 p-2} \\
\vdots \\
u_{p}
\end{array}\right]+\left[\begin{array}{cccc}
u_{2 p-2} & u_{2 p-3} & \cdots & u_{p-1} \\
u_{2 p-3} & u_{2 p-4} & \cdots & u_{p-2} \\
\vdots & \vdots & \ddots & \vdots \\
u_{p-1} & u_{p-2} & \cdots & u_{0}
\end{array}\right]\left[\begin{array}{c}
h_{1} \\
h_{2} \\
\vdots \\
h_{p}
\end{array}\right]=\mathbf{0}} \\
& \Leftrightarrow \boldsymbol{u}+\boldsymbol{U} \boldsymbol{h}=\mathbf{0} \Rightarrow \boldsymbol{h}=-\boldsymbol{U}^{-1} \boldsymbol{u}
\end{aligned}
$$

We may observe that the solution of the coefficients of the pole zero cancellation filter requires the knowledge of the $2 p$ values of the $u_{k}$ sequence. The polynomial $\boldsymbol{h}=\left[\begin{array}{lllll}1 & h_{1} & h_{2} & \cdots & h_{p}\end{array}\right]^{\mathrm{T}}$ has roots $\Delta_{i}(i=1,2, \cdots, p)$. The time locations of the Diracs are then obtained as $t_{i}=t_{0}-\Delta_{i}$. For solution of the amplitudes we may write (7) in matrix/vector form

$$
\begin{aligned}
& {\left[\begin{array}{c}
u_{0} \\
u_{1} \\
\vdots \\
u_{2 p-1}
\end{array}\right]=\left[\begin{array}{cccc}
1 & 1 & \cdots & 1 \\
\Delta_{1} & \Delta_{2} & \cdots & \Delta_{p} \\
\vdots & \vdots & \ddots & \vdots \\
\Delta_{1}^{2 p-1} & \Delta_{2}^{2 p-1} & \cdots & \Delta_{p}^{2 p-1}
\end{array}\right]\left[\begin{array}{c}
a_{1} \\
a_{2} \\
\vdots \\
a_{p}
\end{array}\right]} \\
& \Leftrightarrow \boldsymbol{u}=\boldsymbol{D} \boldsymbol{a} \Rightarrow \boldsymbol{a}=\boldsymbol{D}^{\#} \boldsymbol{u}
\end{aligned}
$$

where \# denotes the pseudoinverse matrix. Now the amplitudes of the impulses are obtained as $A_{i}=a_{i} \mathrm{e}^{\alpha \Delta_{i}}$. To 
summarize, the reconstruction of $p$ impulses requires the knowledge of the $u_{k}(k=0,1, \cdots, 2 p-1)$ sequence. This needs the simultaneous measurement of at least $2 p$ samples from the outputs of the cascaded RC filters. An alternative singular value decomposition (SVD) based null space method is presented in Appendix for the solution of the roots of the pole zero cancellation filter (9).

\subsection{Noise Cancellation}

The above formulation (8)-(13) is valid only in noise free situation. The noise was rejected by the singular value decomposition (SVD) based subspace method. Let us construct a Hankel matrix

$$
\boldsymbol{H}=\left[\begin{array}{cccc}
u_{0} & u_{1} & \cdots & u_{p} \\
u_{1} & u_{2} & \cdots & u_{p+1} \\
\vdots & \vdots & \ddots & \vdots \\
u_{p-1} & u_{p} & \cdots & u_{2 p-1}
\end{array}\right]
$$

where the antidiagonal elements are identical. The singular value decomposition of the matrix $\boldsymbol{H}$ is

$\boldsymbol{H}=\boldsymbol{U} \boldsymbol{\Sigma} \boldsymbol{V}^{\mathrm{T}}$, where $\boldsymbol{U}$ and $\boldsymbol{V}$ are unitary matrices.

$\boldsymbol{\Sigma}$ is a diagonal matrix consisting of the singular values in descending order. We may decompose $\boldsymbol{H}$ as

$$
\begin{aligned}
\boldsymbol{H} & =\left[\begin{array}{ll}
\boldsymbol{U}_{s} & \boldsymbol{U}_{n}
\end{array}\right]\left[\begin{array}{cc}
\boldsymbol{\Sigma}_{s} & \mathbf{0} \\
\mathbf{0} & \boldsymbol{\Sigma}_{n}
\end{array}\right]\left[\begin{array}{ll}
\boldsymbol{V}_{s} & \boldsymbol{V}_{n}
\end{array}\right]^{\mathrm{T}} \\
& =\boldsymbol{U}_{s} \boldsymbol{\Sigma}_{s} \boldsymbol{V}_{s}^{\mathrm{T}}+\boldsymbol{U}_{n} \boldsymbol{\Sigma}_{n} \boldsymbol{V}_{n}^{\mathrm{T}}=\boldsymbol{H}_{s}+\boldsymbol{H}_{n}
\end{aligned}
$$

$\boldsymbol{\Sigma}_{n}$ contains the smallest singular values and hence the matrix $\boldsymbol{H}_{n}$ can be considered to belong to the noise subspace. The matrix $\boldsymbol{H}_{s}$ is then related to the noise free signal subspace. The dimension of the signal subspace can be evaluated by several methods [15]. In this work we plotted the logarithm of the singular values in descending order. The logarithmic singular values form clearly separable linear groups. In all cases the dimension of the signal subspace appeared to be $p$, i.e. the number of impulses. The signal matrix $\boldsymbol{H}_{s}$ is not precisely a Hankel matrix, as some variation occurs in the antidiagonal elements. We reconstructed the noise free Hankel matrix by replacing the antidiagonal elements by their mean values.

\subsection{High-Speed Sampling Method}

In the case the impulse rate is limited so that only one impulse arrives at the cascaded RC network, the reconstruction algorithm simplifies notably and the sampling of the outputs of the first two RC filters is enough to recover the amplitude and time location of the impulse. Based on (6) we obtain

$$
\begin{aligned}
& y_{0}=C_{0} A_{1} \mathrm{e}^{-\alpha \Delta_{1}} \Rightarrow \Delta_{1}=C_{0} y_{1} /\left(C_{1} y_{0}\right) \\
& y_{0}=C_{0} A_{1} \mathrm{e}^{-\alpha \Delta_{1}} \Rightarrow A_{1}=y_{0} \mathrm{e}^{\alpha \Delta_{1}} / C_{0}
\end{aligned}
$$

In practice the outputs of at least three RC filters is advisable to measure, since we may then construct the Hankel matrix $\boldsymbol{H}=\left[\begin{array}{ll}y_{0} & y_{1} \\ y_{1} & y_{2}\end{array}\right]$ for SVD based noise cancellation.

\subsection{Elimination of the Pulse Pile-Up in UWB Transmitter}

The practical UWB transmission protocol consists of wireless RF pulses each containing three impulses (Figure 2). The current reconstruction algorithm $(12,13)$ does not take into account the pulse pile-up effect due to the previous pulses in cascaded RC network. Let us consider the situation in which the UWB pulses are transmitted at constant time intervals $n T, n=0,1, \cdots$ and the parallel sampling of the outputs of the RC network also occurs at $n T$ intervals. By denoting the sampled and normalized outputs by $u_{k}[n], n=0,1,2, \cdots$ and applying (7), we have

$$
\begin{aligned}
\hat{u}_{k}[n+1] & =u_{k}[n+1]-\mathrm{e}^{-\alpha T} \sum_{i=1}^{p} a_{i}\left(\Delta_{i}+T\right)^{k} \\
& \approx u_{k}[n+1]-\mathrm{e}^{-\alpha T} u_{k}[n]
\end{aligned}
$$

contains the output due to the impulses occurring at time interval $[n T,(n+1) T]$ reduced by the pile-up signal due to the previous UWB pulses at time interval $[(n-1) T, n T]$. The approximation in (17) is exact for $k=0$. However, in cases for $k=1,2, \cdots$ the exponential $\mathrm{e}^{-\alpha t}$ is mainly responsible for the descending tail and the approximation error is negligible.

\section{Experimental}

The reconstruction algorithm was tested by an extensive simulation study. The cascaded network comprised eight identical RC filters which were constructed using an ana-
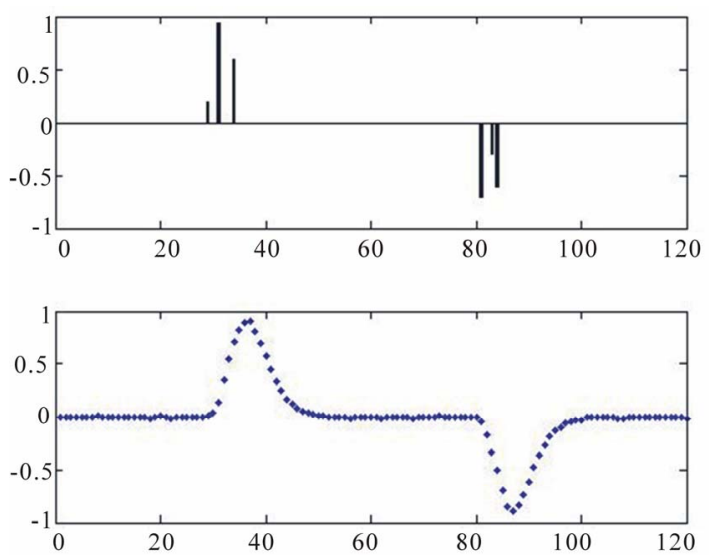

Figure 2. The output signal of the cascaded RC filter network comprising of eight cascaded identical RC circuits. Each UWB pulse consists of three Diracs. 
$\log$ electronic circuit simulator (Spice). The $\alpha$ parameter varied in the range $0.1-0.8$. In the absence of noise the reconstruction algorithm $(12,13)$ recovered the amplitudes and time locations of the impulse train consisting of 1 - 4 Diracs with machine precision. When the random noise was added to the input of the network, the algorithm could reconstruct 1 - 3 Diracs with good accuracy, when the SVD based subspace method was applied to noise cancellation. The error in amplitudes varied between $0.8-1.2$ percent and in time locations $0.3-0.7$ percent. In the case of four sequential Diracs the reconstruction algorithm yielded inaccurate results or totally failed.

We made a preliminary comparison with the multichannel sampling (MCS) arrangement [14] using the Spice simulation toolbox. In the case of $1-3$ sequential impulses the performance of the MCS was clearly poorer than the results obtained by the present $\mathrm{RC}$ filter network in the presence of noise below SNR $<45 \mathrm{~dB}$, but significantly higher at SNR $>45 \mathrm{~dB}$. In the case of four sequential impulses the performance of the MCS was significantly higher compared with the present reconstruction method in all noise levels.

The cascaded RC network was implemented using metallic foil resistors and low-noise capacitors. Eight RC filters were separated by unity gain buffer amplifiers (Figure 1). The outputs of the RC filters were sampled simultaneously with an eight channel differential 12-bit analog-to-digital converter (ADC) equipped with a sample and hold $(\mathrm{S} / \mathrm{H})$ circuit in every channel. For 1 - 3 Diracs the reconstruction error in amplitudes was between $1.1-1.4$ percent and in time locations $0.6-0.9$ percent. For four Diracs the reconstruction error was considerably higher, for amplitudes $2.2-3.4$ percent and for time locations 0.9 - 1.7 percent. In all experimental measurements the SVD based subspace method was used for noise rejection. The high-speed sampling method (16) recovered the amplitudes and time locations of single Diracs with good accuracy using three cascaded RC filters. The amplitude error was between $0.6-1.3$ percent and the error in time locations $0.1-1.1$ percent.

The reconstruction of Diracs using the SVD based null space method (Appendix) gave almost identical results, which are not repeated here. The slight difference is probably due to that the matrices $\boldsymbol{H}$ in (14) and $\boldsymbol{U}$ in (17) are different.

\section{Conclusions}

In our previous work [9] we introduced a novel sampling scheme based on parallel RC filters, where the outputs were measured simultaneously. A disadvantage in the practical construction of the parallel RC network comes from the precise adjustment of the time constants of the
RC circuits. For $N$ parallel RC circuits the $\alpha$ parameter must obey the condition $\alpha_{k}=\alpha_{0}+k \alpha, k=0,1, \cdots, N-1$. On the contrary, in present cascaded network all RC filters and buffer amplifiers are identical and much simpler to implement in integrated VLSI circuits. Compared with the previous FRI methods, which are based on the sequential sampling, the outputs of the parallel RC filters are sampled only once per UWB pulse, which contains a restricted number of impulses (Figure 2). This eliminates the need for high frequency analog-to-digital converters (ADCs), since the UWB pulse repetition rate can be adjusted to match the ADC's performance.

The formulation of the reconstruction algorithm was warranted by extensive simulation study using Spice toolbox. In the presence of noise the SVD based noise cancellation method had to be applied. The computational complexity of the SVD algorithm is $O\left(N^{3}\right)$, which restricts the real-time applications of the present method to a relatively low transmission rate. However, since the information is coded to both the amplitude and the time locations of the Diracs, the number of transmitted pulses can be significantly lower compared with the conventional UWB methods. As an advantage lower number of transmitted pulses reduces the RF radiation load.

We showed in Spice simulation study that in noise free conditions the $p$ Diracs can be reconstructed from sampling the outputs of the $2 p$ cascaded identical RC filters. In the presence of noise this theoretical " $2 p$ rule" is not valid and it seems evident that preferable " $2 p+1$ rule" should be applied.

The high-speed method (16) suits best for reconstruction of single Diracs, whose amplitudes are quantized to only a few levels. For example the impulses with 15 discrete levels can be produced by a 4-bit digital-to-analog converter, which the method reproduces perfectly.

In MCS arrangement the signal is modulated by a set of sinusoidal waveforms, followed by a bank of integrators [14]. The MCS produces Fourier series coefficients, which enable to reconstruct the input signal. However, the MCS is much more complex compared to the circuit consisting of the cascaded identical RC filters. The Spice simulation study revealed that the MCS outperforms the present approach at high noise level, SNR over $45 \mathrm{~dB}$, but inferiors at lower noise environment. To the best of our knowledge the hardware for the implementation of the MCS is not yet available. Hence, in this moment this figure is based only on a simulation study.

In most of the UWB devices information is transmitted by monocycle Gaussian pulses. The FCC restricted the UWB frequency band between $3.1-10.6 \mathrm{GHz}$ in year 2002 [16]. The Gaussian pulse train does not meet this constraint and other pulse shapes have been introduced to meet the FCC criteria, e.g. the family of the orthogonal 
UWB pulse waveforms $[17,18]$. The fast digital-to-analog converters (DACs) can produce UWB pulses, where the information is coded via orthogonal base vectors and reproduced by matrix/vector computation.

In this work we have concentrated on the UWB communication device, which transmits pulses consisting of most 1 - 3 impulses. The information is coded to the amplitudes and time locations of the Diracs. Such pulse generators are relatively easy to implement in VLSI [19]. Following [18] the impulse train can be designed so that its spectral density spectrum coincides with the FCC criteria.

\section{Acknowledgements}

This work was supported by the National Technology Agency of Finland (TEKES).

\section{REFERENCES}

[1] P. Marziliano, "Sampling Innovations," Ph.D. Dissertation, Swiss Federal Institute of Technology, Lausanne, 2001.

[2] M. Vetterli, P. Marziliano and T. Blu, "Sampling Signals with Finite Rate of Innovation," IEEE Transactions on Signal Processing, Vol. 50, No. 6, 2002, pp. 1417-1428. doi:10.1109/TSP.2002.1003065

[3] I. Maravic and M. Vetterli, "Sampling and Reconstruction of Signals with Finite Rate of Innovation in the Presence of Noise," IEEE Transactions on Signal Processing, Vol. 53, No. 8, 2005, pp. 2788-2805. doi:10.1109/TSP.2005.850321

[4] P. Marziliano, M. Vetterli and T. Blu, "Sampling and Exact Reconstruction of Band Limited Signals with Additive Shot Noise," IEEE Transactions on Information Theory, Vol. 52, No. 5, 2006, pp. 2230-2233. doi:10.1109/TIT.2006.872844

[5] Jovanovic and B. Beferull-Lozano, "Oversampled A/D Conversion and Error-Rate Dependence of Nonband Limited Signals with Finite Rate of Innovation," IEEE Transactions on Signal Processing, Vol. 54, No. 6, 2006, pp. 2140-2154. doi:10.1109/TSP.2006.874363

[6] J. Haupt and R. Nowak, "Signal Reconstruction from Noisy Random Projections," IEEE Transactions on Information Theory, Vol. 52, No. 9, 2006, pp. 4036-4048. doi:10.1109/TIT.2006.880031

[7] Y. C. Eldar and M. Unser, "Nonideal Sampling and Interpolation from Noisy Observations in Shift-Invariant Spaces," IEEE Transactions on Signal Processing, Vol. 54, No. 7, 2006, pp. 2636-2651. doi:10.1109/TSP.2006.873365
[8] P. L. Dragotti, M. Vetterli and T. Blu, "Sampling Moments and Reconstructing Signals of Finite Rate of Innovation: Shannon Meets Strang-Fix," IEEE Transactions on Signal Processing, Vol. 55, No. 5, 2007, pp. 17411757. doi:10.1109/TSP.2006.890907

[9] H. Olkkonen and J. T. Olkkonen, "Measurement and Reconstruction of Impulse Train by Parallel Exponential Filters," IEEE Signal Processing Letters, Vol. 15, 2008, pp. 241-244. doi:10.1109/LSP.2008.916998

[10] J. T. Olkkonen and H. Olkkonen, "Reconstruction of Wireless UWB Pulses by Exponential Sampling Filter," Wireless Sensor Network, Vol. 2, No. 6, 2010, pp. 462466. doi:10.4236/wsn.2010.26057

[11] J. T. Olkkonen and H. Olkkonen, "Sampling and Reconstruction of Piecewise Constant Signals by Parallel CRNetwork," IEEE Transactions on Circuits and Systems II, Vol. 55, No. 11, 2008, pp. 1159-1162. doi:10.1109/TCSII.2008.2002568

[12] J. T. Olkkonen and H. Olkkonen, "Sampling and Reconstruction of Zero-Order Hold Signals by Parallel RC Filters," Wireless Engineering and Technology, Vol. 2, No. 3, 2011, pp. 153-156. doi:10.4236/wet.2011.23022

[13] H. Olkkonen and J. T. Olkkonen, "Measurement and Reconstruction of Transient Signals by Parallel Exponential Filters," IEEE Transactions on Circuits and Systems II, Vol. 57, No. 6, 2010, pp. 426-429. doi:10.1109/TCSII.2010.2048375

[14] K. Gedalyahu, R. Tur and Y. C. Eldar, "Multichannel Sampling of Pulse Streams at the Rate of Innovation," IEEE Transactions on Signal Processing, Vol. 59, No. 4, 2011, pp. 1491-1504. doi:10.1109/TSP.2011.2105481

[15] E. Biglieri and K. Yao, "Some Properties of Singular Value Decomposition and Their Applications to Digital Signal Processing," Signal Processing, Vol. 18, 1989, pp. 277-289. doi:10.1016/0165-1684(89)90039-X

[16] Y. P. Nakache and A. F. Molisch, "Spectral Shaping of UWB Signals for Time-Hopping Impulse Radio," IEEE Journal of Selected Areas of Communication, Vol. 24, No. 4, 2006, pp. 738-744. doi:10.1109/JSAC.2005.863817

[17] B. Parr, B. Cho, K. Wallace and Z. Ding, "A Novel U1tra-Wideband Pulse Design Algorithm," IEEE Communications Letters, Vol. 7, No. 5, 2003, pp. 219-221. doi:10.1109/LCOMM.2003.812167

[18] H. Olkkonen and J. T. Olkkonen, "Design of Orthogonal UWB Pulse Waveform for Wireless Multi-Sensor Applications," Wireless Sensor Network, Vol. 2, No. 11, 2010, pp. 850-853. doi:10.4236/wsn.2010.211102

[19] M. Miao and C. Nguyen, "On the Development of an Integrated CMOS-Based UWB Tunable-Pulse Transmit Module," IEEE Transactions on Microwave Theory and Techniques, Vol. 54, No. 10, 2006, pp. 3681-3687. doi:10.1109/TMTT.2006.882874 


\section{Appendix}

\section{SVD Based Null Space Solution}

Let us write the convolution (11) in the matrix/vector form

$$
\begin{aligned}
& {\left[\begin{array}{cccc}
u_{2 p-1} & u_{2 p-2} & \cdots & u_{p-1} \\
u_{2 p-2} & u_{2 p-3} & \cdots & u_{p-2} \\
\vdots & \vdots & \ddots & \vdots \\
u_{p} & u_{p-1} & \cdots & u_{0}
\end{array}\right]\left[\begin{array}{c}
h_{0} \\
h_{1} \\
\vdots \\
h_{p}
\end{array}\right]=\mathbf{0}} \\
& \Leftrightarrow \boldsymbol{U h}=\mathbf{0}
\end{aligned}
$$

In the following we describe the singular value decomposition (SVD) based null space solution of the unknown vector $\boldsymbol{h}$ in (18). The SDV of the $\boldsymbol{U}$ matrix in (18) yields

$$
\boldsymbol{U}=\boldsymbol{L} \boldsymbol{\Sigma} \boldsymbol{R}^{\mathrm{T}}
$$

where matrices $\boldsymbol{L}=\left[\begin{array}{llll}\boldsymbol{l}_{1} & \boldsymbol{l}_{2} & \cdots & \boldsymbol{l}_{p+1}\end{array}\right]$ and $\boldsymbol{R}=\left[\begin{array}{llll}\boldsymbol{r}_{1} & \boldsymbol{r}_{2} & \cdots & \boldsymbol{r}_{p+1}\end{array}\right]$ contain the left and right singular vectors (column vectors) and matrix

$\boldsymbol{\Sigma}=\operatorname{diag}\left\{\begin{array}{cccc}\sigma_{1} & \sigma_{2} & \cdots & \sigma_{p+1}\end{array}\right\}$ the singular values in de- scending order. Matrices $\boldsymbol{L}$ and $\boldsymbol{R}$ are unitary, i.e. $\boldsymbol{L}^{\mathrm{T}} \boldsymbol{L}=\boldsymbol{I}$ and $\boldsymbol{R}^{\mathrm{T}} \boldsymbol{R}=\boldsymbol{I}$, where $\boldsymbol{I}$ is the identity matrix. Applied to (19) we obtain $\boldsymbol{U R}=\boldsymbol{L} \boldsymbol{\Sigma}$, which yields

$$
\boldsymbol{U}\left[\boldsymbol{r}_{1} \boldsymbol{r}_{2} \cdots \boldsymbol{r}_{p+1}\right]=\left[\sigma_{1} \boldsymbol{l}_{1} \sigma_{2} \boldsymbol{l}_{2} \cdots \sigma_{p+1} \boldsymbol{l}_{p+1}\right]
$$

Finally we may write

$$
\boldsymbol{U} \boldsymbol{r}_{k}=\sigma_{k} \boldsymbol{l}_{k}
$$

for $k=1,2, \cdots, p+1$. Equation (21) forms the basis for the SVD based null space method. By searching very small singular value $\sigma_{k} \geq 0$, the right singular vector $\boldsymbol{r}_{k}$ equals vector $\boldsymbol{h}$ in (18) yielding the solution for the roots of the pole cancellation filter (9). In the presence of noise the dimensions of the $\boldsymbol{L}$ and $\boldsymbol{R}$ matrices should be selected so that there appears only one tiny singular value. This can be also achieved by zeroing the rest of the tiny singular values. It should be pointed out that the SVD based null space method does not yield the coefficients of the pole zero cancellation filtering (9) in normalized form, i.e. $h_{0} \neq 1$. However, we may apply post normalization as $h_{k} \leftarrow h_{k} / h_{0}$ for $k=0,1, \cdots, p$. 